\title{
Effect of ketoconazole-mediated CYP3A4 inhibition on clinical pharmacokinetics of panobinostat (LBH589), an orally active histone deacetylase inhibitor
}

\author{
Paul Hamberg • Margaret M. Woo $\cdot$ Lin-Chi Chen • Jaap Verweij • \\ Maria Grazia Porro • Lily Zhao - Wenkui Li • Diane van der Biessen • \\ Sunil Sharma $\cdot$ Thomas Hengelage $\cdot$ Maja de Jonge
}

Received: 4 April 2011/Accepted: 9 June 2011/Published online: 26 June 2011

(C) The Author(s) 2011. This article is published with open access at Springerlink.com

\begin{abstract}
Purpose Panobinostat is partly metabolized by CYP3A4 in vitro. This study evaluated the effect of a potent CYP3A inhibitor, ketoconazole, on the pharmacokinetics and safety of panobinostat.

Methods Patients received a single panobinostat oral dose on day 1 , followed by 4 days wash-out period. On days 59, ketoconazole was administered. On day 8, a single panobinostat dose was co-administered with ketoconazole. Panobinostat was administered as single agent three times a week on day 15 and onward.
\end{abstract}

Previously presented in part at the Annual Meeting of the American Society of Clinical Oncology 2008 (oral presentation).

ClinicalTrials.gov identifier: NCT 00503451.

P. Hamberg $(\bowtie) \cdot$ J. Verweij · D. van der Biessen · M. de Jonge Department of Medical Oncology, Erasmus University

Medical Center, Daniel den Hoed Cancer Center,

P.O. Box 5201, 3008 AE Rotterdam, The Netherlands

e-mail: a.hamberg@erasmusmc.nl

M. M. Woo $\cdot$ L. Zhao

Novartis Pharmaceutical Corporation, Florham Park, NJ, USA

L.-C. Chen $\cdot$ S. Sharma

Nevada Cancer Institute, Las Vegas, NV, USA

M. G. Porro - T. Hengelage

Novartis Pharma AG, Basel, Switzerland

W. Li

Novartis Pharmaceuticals, East Hanover, NJ, USA

Present Address:

P. Hamberg

Sint Franciscus Gasthuis, Rotterdam, The Netherlands
Results In the presence of ketoconazole, there was 1.6and 1.8-fold increase in $C_{\max }$ and AUC of panobinostat, respectively. No substantial change in $T_{\max }$ or half-life was observed. No difference in panobinostat-pharmacokinetics between patients carrying $C Y P 3 A 5^{*} 1 / * 3$ and $C Y P 3 A 5 * 31$ $* 3$ alleles was observed. Most frequently reported adverse events were gastrointestinal related. Patients had asymptomatic hypophosphatemia (64\%), and urine analysis suggested renal phosphate wasting.

Conclusions Co-administration of panobinostat with CYP3A inhibitors is feasible as the observed increase in panobinostat PK parameters was not considered clinically relevant. Considering the variability in exposure following enzyme inhibition and the fact that chronic dosing of panobinostat was not studied with CYP3A inhibitors, close monitoring of panobinostat-related adverse events is necessary.

Keywords Panobinostat - LBH589 - Histone deacetylase inhibitor · CYP3A

\section{Introduction}

Histone deacetylases (HDACs) are enzymes controlling the acetylation state of lysine protein residues, notably those contained in the N-terminal portions of the core histones, and are functionally counteracted by histone acetylases. The balance between these two enzyme groups controls cell cycle progression, cell survival and differentiation. In neoplastic cells, the abundance of deacetylated histones is usually associated with DNA hypermethylation and gene silencing [1,2]. As a new class of chemotherapeutic agents, HDAC inhibitors (HDACi) have demonstrated potent anticancer activities in preclinical studies and are currently 
in various stages of clinical development. The molecular basis of the anticancer effects of HDACi goes beyond the inhibition of histone acetylation alone. Over the last several years, a growing list of non-histone targets important in the regulation of cell proliferation, cell death and cell migration have been identified, including p53, NF- $\kappa \mathrm{B}, \mathrm{Ku} 70$, $\alpha$-tubulin and Hsp90 [3].

HDACs are grouped based on their catalytic mechanism: class 1 (HDACs 1,2,3 and 8), class II a/b (HDACs 4,5,6,7,9 and 10), class III (sirtuin enzymes) and Class IV (HDAC 11) [2]. Inhibitors of HDAC class I as well as class II are arbitrarily called pan-deacetylase inhibitors (panDACi).

Panobinostat (LBH589) is a pan-DACi, which belongs to a structurally novel cinnamic hydroxamic acid class of compounds [4].

It is one of the most potent class I/II pan-DACi in vitro and has shown antitumor activity in a wide variety of tumor xenograft models. Reports from phases I-II studies have demonstrated signs of clinical activity of panobinostat in patients with cancer, e.g., in cutaneous T-cell lymphoma, Hodgkin's or non-Hodgkin's lymphoma, prostate cancer, acute myeloid leukemia, multiple myeloma and breast cancer [5-7]. Phase II studies are ongoing in patients with hematological malignancies and solid tumors. Thrombocytopenia and fatigue were the dose-limiting toxicities in the phase I study after oral administration of panobinostat. Other major toxicities were nausea and vomiting.

Panobinostat is metabolized via multiple pathways including reduction, hydrolysis, oxidation and glucuronidation. The oxidative metabolism of panobinostat is mediated mainly by human cytochrome P450 CYP3A4 (7098\%) with minor involvement of CYP2D6 and CYP2C19.

CYP3A is a predominant family of CYP enzymes, which makes it one of the most important drug-metabolizing enzymes. CYP3A is expressed primarily in liver and small intestine [8]. Since hepatic CYP3A4 has been estimated to metabolize close to $50 \%$ of currently used drugs, as well as endogenous and exogenous steroids, it is evident that anticancer agents including panobinostat given in combination with drugs that are CYP3A4 inhibitors are likely to result in clinical toxicity. Indeed, based on in silico drug-drug interaction (DDI) modeling and simulation, ketoconazole, a strong CYP3A4 inhibitor, was predicted to increase panobinostat AUC by 1.3-2 fold. Thus, a decision was made to study panobinostat at the lowest efficacious dose at $20 \mathrm{mg} /$ day to assess the clinical drugdrug interaction potential. The study was designed to determine the effect of a strong CYP3A inhibitor on the systemic exposure to panobinostat, in order to assess whether or not co-administration with a CYP3A inhibitor can be allowed in patients if medically necessary during treatment with panobinostat.
The primary and secondary objectives of this study were to investigate the effect of multiple doses of ketoconazole on the pharmacokinetics of a single dose of panobinostat and safety, respectively.

\section{Patients and methods}

\section{Patient selection}

Patient selection was performed at the phase I outpatient department. Patients with histologically or cytologically confirmed advanced or metastatic solid tumors for whom no standard therapy was available and with an Eastern Cooperative Oncology Group (ECOG) performance status $\leq 2$ were eligible. Since clinical responses were observed in phase I/II trials with panobinostat, the selection of this phase I patients population with no other therapeutic options was considered appropriate for this study where a prolonged treatment with a potentially active drug was offered to these patients, although, based on tumor characteristics no potential benefit was anticipated beforehand. The serum potassium, total corrected calcium, magnesium and phosphorus had to be above the lower limit of normal. QTc had to be $\leq 450 \mathrm{~ms}$. Concomitant treatment with CYP3A inhibitors and medications which increase the risk of Torsades de Point was not allowed. Significant cardiac disease or other concurrent uncontrolled medical conditions were exclusionary criteria. The study was designed and conducted under the appropriate institutional review boards' approvals and in accordance with the principles embodied in the Declaration of Helsinki. Written informed consent was obtained from each participant. This study is registered as NCT00503451 (http://www.ClinicalTrials.gov).

\section{Study design}

Patients received single-agent panobinostat at $20 \mathrm{mg}$ orally after at least $2 \mathrm{~h}$ fasting on day 1 and single-agent ketoconazole once daily at $400 \mathrm{mg}$ on days 5-9 (Fig. 1). On day 8 , panobinostat was administered $1 \mathrm{~h}$ after ketoconazole. If at interim analysis after evaluation of 12 patients any of the four parameters $\left(\mathrm{AUC}_{0-24}, \mathrm{AUC}_{0-\text { tlast }}, \mathrm{AUC}_{0-\infty}\right.$ and $C_{\max }$ ) had the paired t statistic (calculated based on log-transformed data) falling outside of $(-1.98405$, 1.98405), then the recruitment was to be stopped. If not, the trial was to proceed until 16 patients completed the study. The study design also stipulated that, while completer status for 12 patients was being confirmed, enrollment was not halted as long as there were no safety concerns.

All patients enrolled before this interim analysis were to proceed to the extension phase regardless of the interim result. From day 15 onward, the extension phase of the 


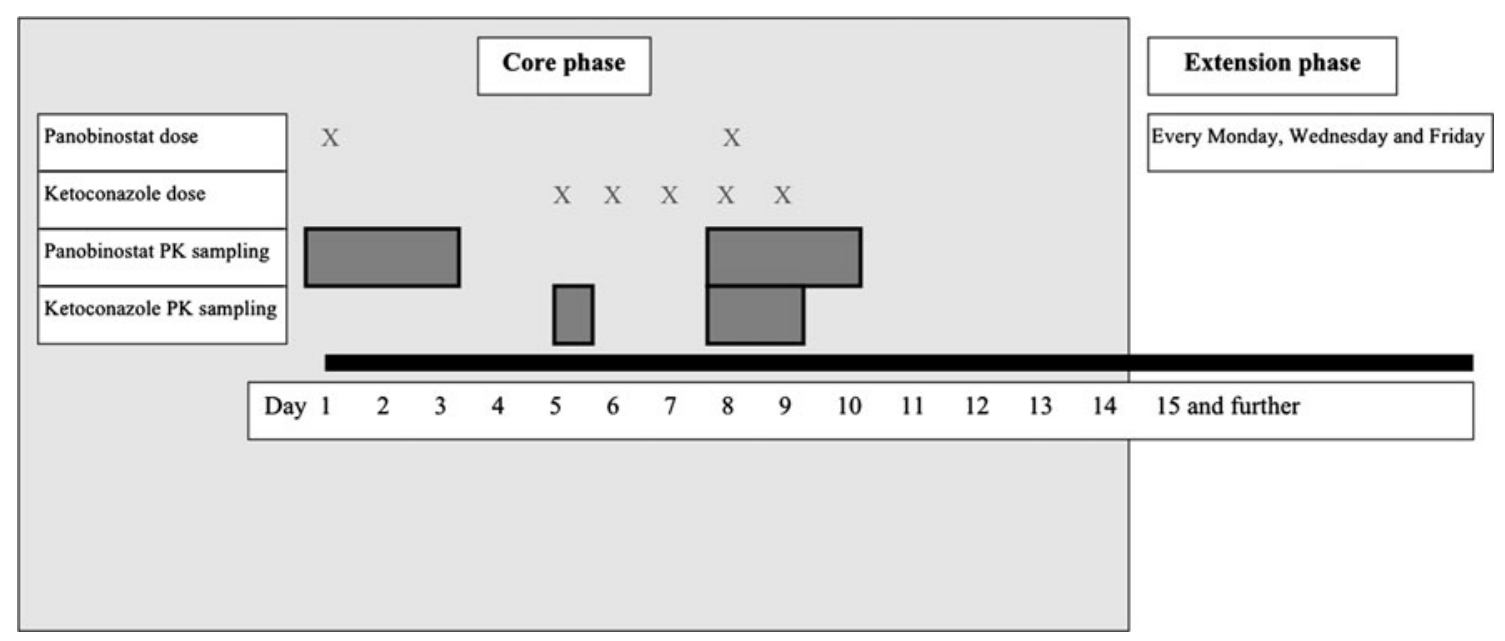

Fig. 1 Study design. Time points of dosing and pharmacokinetic sampling

study started in which patients received single-agent $20 \mathrm{mg}$ oral panobinostat each Monday, Wednesday and Friday (MWF) until disease progression, patient withdrawal of consent or unacceptable toxicity. Toxicity was evaluated using the Common Terminology Criteria for Adverse Events (CTCAE) v 3.0.

\section{Safety assessments}

Close monitoring by evaluating signs and symptoms, physical examination, laboratory assessments and ECGs was performed during the trial. Patients had scheduled visits on days $1,2,3,5,8,9,10$ and 15 . Laboratory assessments were scheduled at days 1, 5, 8, 9, 10 and 15 . Twelve-lead ECGs were scheduled as follows: predose day 1 and at 1.5, 3, 6, 24 and $48 \mathrm{~h}$ post-dose; preketoconazole dose on day 5 and at 1.5 and $3 \mathrm{~h}$ post-dose; preketoconazole on day 8 and 1.5, 3, 6, 24 and $48 \mathrm{~h}$ post-dose panobinostat; and predose on day 15 . All ECGs were taken at least in triplicate separated by $2-5 \mathrm{~min}$ and were reviewed independently at a central laboratory (eRT, Bridgewater, NJ). During the extension phase, single ECGs were performed weekly as were laboratory assessments. Bazett's formula was used to correct QT intervals for heart rate.

\section{Pharmacokinetic evaluation}

During cycle 1 at days 1 and 8 , patients had serial blood sampling collections for pharmacokinetic evaluation of panobinostat. Blood samples ( $3 \mathrm{ml} / \mathrm{sample})$ were collected in tubes containing sodium heparin prior to panobinostat dose and $0.5,1,1.5,2,2.5,3,4,6,8,24$ and 48 h postdose. Samples were centrifuged at $800 \mathrm{~g}$ for $15 \mathrm{~min}$ at $4^{\circ} \mathrm{C}$ to yield plasma within 60 min after blood collection and stored below $-60^{\circ} \mathrm{C}$ until analyses.

Blood samples $(3 \mathrm{ml})$ for ketoconazole concentration determinations were collected predose and $2 \mathrm{~h}$ post-dose of ketoconazole on days 5, 8 and 9 (Fig. 1).

Panobinostat is analyzed in human plasma samples using a specific LC-MS/MS method with the lower limit of quantification (LLOQ) of $0.5 \mathrm{ng} / \mathrm{ml}$ and a dynamic range of $0.5-500 \mathrm{ng} / \mathrm{ml}$ of the analyte using $0.1 \mathrm{ml}$ sample volume. A semi-automated protein precipitation extraction of human plasma samples was used to separate panobinostat from human plasma protein. The obtained sample extracts were evaporated to dryness, reconstituted with $10 \%$ aqueous acetonitrile (containing $0.2 \%$ formic acid) and analyzed by LC-MS/MS on a Sciex API3000 or a Sciex API4000 tandem mass spectrometer. A Waters Xbridge C8 column $(2.5 \mu \mathrm{m}$ particle size, $50 \times 2.1 \mathrm{~mm})$ was employed in reversed-phase chromatography with gradient elution using $10 \%$ acetonitrile in water (containing $0.1 \%$ formic acid and $0.1 \%$ acetic acid, mobile phase A) and $90 \%$ acetonitrile in water (containing $0.25 \%$ formic acid and $0.25 \%$ acetic acid, mobile phase B) at a flow rate of $0.3 \mathrm{ml}: / \mathrm{min}$. The assay was linear from 0.5 to $500 \mathrm{ng} / \mathrm{ml}$ with the bias (\%) and $\mathrm{CV}(\%)$ values of the $\mathrm{QC}$ sample results ranging from -0.7 to $0.7 \%$ and 2.3 to $11.6 \%$, respectively, based on the within-study validation during the sample analysis.

For determination of ketoconazole in human plasma, a $100 \mu \mathrm{l}$ aliquot of each sample (Standards, QC samples, study samples, blanks, etc.,) was pipetted into the appropriate well in a 96-deep-well plate. A $100 \mu \mathrm{l}$ aliquot of the internal standard working solution $(250 \mathrm{ng} / \mathrm{ml}$ in $50 \%$ acetonitrile, v/v) was added to all wells except for the blanks, to which a $100 \mu \mathrm{l}$ aliquot of $50 \%$ acetonitrile was 
added. A $400 \mu \mathrm{l}$ aliquot of acetonitrile was added to each well, the plate covered, vortexed for approximately $10 \mathrm{~min}$ and centrifuged at approximately 4,000 rpm for approximately $10 \mathrm{~min}$ at room temperature. Using a TomTec device, a $50 \mu \mathrm{l}$ aliquot of sample extract was transferred to a clean 96-deep-well plate, followed by an addition of $450 \mu \mathrm{l}$ of $30 \%$ acetonitrile in water (v/v). After a brief vortex mixing, a $10 \mu \mathrm{l}$ aliquot was injected onto the HPLC system connected to a Sciex API5000 mass spectrometer. The assay was linear from 50.0 to $15,000 \mathrm{ng} / \mathrm{ml}$ with the bias (\%) and $\mathrm{CV}(\%)$ values of the QC sample results ranging from -4.2 to $3.3 \%$ and 6.0 to $8.6 \%$, respectively, based on the within-study validation during the sample analysis.

Panobinostat plasma concentration-time curve following each dose from individual patient was used to calculate pharmacokinetic parameters that included $C_{\max }, T_{\max }$ and $\mathrm{AUC}_{0-\infty}$. PK parameters were obtained using non-compartmental methods implemented in WinNonlin Pro software (Version 5.01, Pharsight Corporation, Mountain View, CA, USA). $C_{\max }$ and $T_{\max }$ were obtained by visual inspection of the concentration-time curve. $\mathrm{AUC}_{0-\infty}$ was calculated using the linear up/log down method up to the last measured concentrations and the additional area estimated from that concentration and the value of apparent terminal half-life estimated for the targeted administration. The apparent terminal half-life was estimated using the best-fit variables of a single exponential to the log-linear portion of the plasma concentration-time curve using nonweighted linear regression.

Ratio was calculated using the following formula: ([PKparameter $\left.]_{\text {panobinostat }+ \text { ketoconazole }} /[\text { PK-parameter }]_{\text {panobinostat }}\right)$.

Pharmacogenetic evaluation

Blood samples (1.5 ml in EDTA-containing tubes) were obtained at baseline for genotyping analysis of CYP3A4*1B, CYP3A5*2, *3, *6 and *7 and performed using AmpliChip CYP450 at Epidauros, Bernried, Germany. DNA preparation and sequencing followed the method described by Sanger et al. (16), using commercial kits provided by Applied Biosystems. Wild-type allele CYP3A4*1A is defined as the exclusion of allele CYP3A4*1B. Wild-type allele CYP3A5*1 is defined as the exclusion of alleles $* 2, * 3, * 6$ and $* 7$.

Sample size calculation and statistical considerations

Pharmacokinetic evaluations to determine the influence of the potent CYP3A inhibitor ketoconazole on the pharmacokinetics of panobinostat were conducted during cycle 1 at days 1-14. A sample size of 16 would have provided enough power $(82.4 \%)$ to detect a minimum increase of $30 \%$ (ketoconazole plus panobinostat vs. panobinostat alone) with an alpha level of 0.1. Since strong interaction between ketoconazole and panobinostat is possible, a ratio higher than 1.3 (ketoconazole plus panobinostat vs panobinostat alone) might be observed. Thus, a drug-drug interaction (DDI) could become apparent with fewer patients, and stopping if this occurs avoids unnecessary exposure of patients to study medications. An interim analysis of PK data was carried out when $75 \%$ of anticipated patients (12 completed patients out of 16 planned completers) were enrolled and had completed the core phase.

The number of 12 patients was chosen with two considerations. A sample size of 12 is generally accepted to evaluate safety [9], and secondly, it provided reasonable power for stopping at interim. The O'Brien-Fleming method was used to calculate the boundaries at interim and final analyses [10].

A linear mixed effects model was fit to the log-transformed PK parameters $\left(C_{\max }\right.$ and $\mathrm{AUC}_{0-24}, \mathrm{AUC}_{0-\mathrm{t}}$ and $\mathrm{AUC}_{0-\infty}$ ). Included in the model was treatment (ketoconazole + panobinostat or panobinostat alone) as fixed effects and patient as a random effect. For the DDI analysis, the combination treatment (ketoconazole + panobinostat) was the test and panobinostat alone the reference. Proper contrasts were used to estimate the treatment difference (i.e., ketoconazole + panobinostat vs. panobinostat alone). The point estimate of the treatment difference and the corresponding $90 \%$ confidence intervals were calculated and back-transformed to obtain the point estimate and $\mathrm{CI}$ on the linear scale for the ratio of geometric means of the test as compared with the reference. The median, minimum and maximum of $T_{\max }$ difference between test and reference were also presented.

\section{Results}

The interim analysis resulted in early stop of the study. Fourteen patients were enrolled, all of them evaluable for pharmacokinetic and safety assessment. Thirteen patients continued in the extension phase. One patient discontinued the study in the core phase due to mild increase in serum creatinine. Patient characteristics are shown in Table 1. All 14 patients have discontinued the study (10 for disease progression, three for adverse events, one for on-treatment death unrelated to study drug due to myocardial infarction). The mean duration of exposure to panobinostat was 140 days (median [range]: 59 [8, 899] days).

Pharmacokinetic evaluation

Pharmacokinetic parameters are shown in Table 2. No residual panobinostat was detectable prior to the day 8 dose 
Table 1 Demographics, tumor types and prior treatment

\begin{tabular}{lc}
\hline Age, median (range) in years & $59(41-74)$ \\
Male & $9(64 \%)$ \\
Female & $5(36 \%)$ \\
Caucasian & $14(100 \%)$ \\
ECOG PS & \\
0 & $3(29 \%)$ \\
1 & $9(57 \%)$ \\
2 & $2(14 \%)$ \\
Primary tumor & $3(21 \%)$ \\
Colorectal & $2(14 \%)$ \\
Pancreas & $1(7 \%)$ \\
Breast & $1(7 \%)$ \\
Prostate & $1(7 \%)$ \\
Salivary gland & $1(7 \%)$ \\
Thyroid & $1(7 \%)$ \\
Head and neck & $4(29 \%)$ \\
Other primary tumor sites & \\
Prior antineoplastic systemic & \\
treatment & \\
Yes & \\
No &
\end{tabular}

(the start of the combination panobinostat plus ketoconazole). In the presence of ketoconazole, peak plasma concentration of panobinostat $\left(C_{\max }\right)$ increased from 18.52 to $29.98 \mathrm{ng} / \mathrm{ml}$, and the area-under-the-curve $0_{0-\infty}\left(\mathrm{AUC}_{0-\infty}\right)$ rose from 133 to $220.7 \mathrm{ng} \mathrm{h} / \mathrm{ml}$, generating ratios of 1.6 (90\% CI 1.2-2.2) and 1.8 (90\% CI 1.5-2.2), respectively. No substantial change in time to maximum concentration $\left(T_{\max }\right)$ or half-life was observed. Individual geometric mean ratios of panobinostat $C_{\max }$ and AUC are shown in

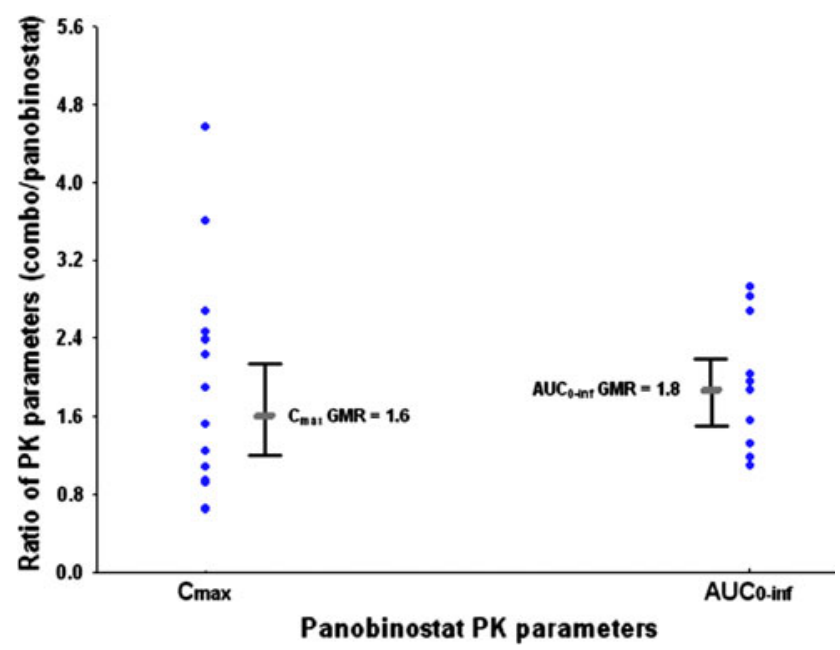

Fig. 2 Individual patient panobinostat PK parameters ratios (panobinostat + ketaconazole/panobinostat) and geometric mean ratio (GMR; value as represented by vertical line) with $90 \%$ CI (bars)

Fig. 2. Of note, in a limited number of patients, a higher than twofold increase in $C_{\max }$ and AUC of panobinostat was observed in the presence of ketoconazole.

Pharmacogenetic evaluation

Genotype analysis of CYP3A4*1B, CYP $3 \mathrm{~A} 5 * 2, * 3, * 6$ and $* 7$ was performed and available at baseline from all 14 patients. All patients in this study were Caucasians. Fourteen patients were homozygous for the wild-type CYP3A4*1A genotype. Eleven patients were homozygous for the CYP3A5*3 genotype, and 3 patients were heterozygous for the CYP $3 \mathrm{~A} 5 * 1 / * 3$ genotype.

There is no apparent difference in panobinostat $C_{\max }$ or AUC value between patients carrying $C Y P 3 A 5^{*} 1 / * 3$ and CYP $3 A 5 * 3 / * 3$ alleles.

Table 2 Analysis of pharmacokinetic parameters of panobinostat administered alone or concomitantly with ketoconazole

\begin{tabular}{llll}
\hline Pharmacokinetic parameter (unit) & $\begin{array}{l}\text { Panobinostat alone } \\
(n=14)^{\mathrm{a}}\end{array}$ & $\begin{array}{l}\text { Panobinostat }+ \text { ketoconazole } \\
(n=14)^{\mathrm{a}}\end{array}$ & $\begin{array}{l}\text { Ratio }(90 \% \mathrm{CI})^{\mathrm{b}} \\
C_{\max }(\mathrm{ng} / \mathrm{ml})\end{array}$ \\
$\mathrm{AUC}_{0-\infty}(\mathrm{ng} \mathrm{h} / \mathrm{ml})$ & $18.52(42.6)$ & $29.98(93.3)$ & $1.6(1.2-2.2)$ \\
$T_{\max }(\mathrm{h})$ & $133.0(39.9)^{\mathrm{c}}$ & $220.7(54.6)^{\mathrm{d}}$ & $0.0(-2.5$ to 3.0$)$ \\
$\mathrm{T} 1 / 2(\mathrm{~h})$ & $1.00(0.5-4.0)$ & $1.00(0.5-6.0)$ & $13.4(7.2-20.9)^{\mathrm{d}}$ \\
$\mathrm{CL} / \mathrm{F}(\mathrm{L} / \mathrm{h})$ & $14.0(6.5-17.2)^{\mathrm{c}}$ & 90.6 & \\
\hline
\end{tabular}

\footnotetext{
${ }^{a}$ Values are median (range) for $T_{\max }$ and $\mathrm{T} 1 / 2$ and geometric mean (CV\%) for all other parameters

${ }^{\mathrm{b}}$ Statistical analysis performed on the primary endpoint pharmacokinetic parameters to show geometric mean ratios for $C_{\text {max }}$ and $\mathrm{AUC}_{0-\infty}$ and median (minimum and maximum difference) for $T_{\max }$

c Data available on $n=11$

d Data available on $n=12$
} 
Table 3 ECG changes during the core phase of the trial

\begin{tabular}{lccc}
\hline & Period $1^{\mathrm{a}}$ & Period 2 $^{\mathrm{a}}$ & Period $^{\mathrm{a}}$ \\
\hline QTcB increase $>30 \mathrm{~ms}$ & $4(29 \%)$ & $3(21 \%)$ & $5(36 \%)$ \\
QTcB increase $>60 \mathrm{~ms}$ & 0 & 0 & 0 \\
New QTcB $>450 \mathrm{~ms}$ & $3(21 \%)$ & $2(14 \%)$ & $2(14 \%)$ \\
Sinus bradycardia & 0 & $1(7 \%)$ & 0 \\
Sinus tachycardia & 0 & 0 & $2(14 \%)$ \\
ST segment depression & 0 & 0 & $2(14 \%)$ \\
$T$ wave changes & $2(14 \%)$ & $1(7 \%)$ & $2(14 \%)$ \\
Ectopic activity & $2(14 \%)$ & $1(7 \%)$ & $2(14 \%)$ \\
\hline
\end{tabular}

In all rows, the number of patients is given (the percentage of patients between brackets; $N=14$ in all periods)

a Period 1 from day 1 until the first dose of ketoconazole; period 2 from first dose of ketoconazole until concomitant administration of panobinostat with ketoconazole (on day 8); and period 3 from the time point of this combination until day 15

\section{Safety results}

In order to determine whether the combination of panobinostat plus ketoconazole resulted in additional toxicity, the core phase of the study was subdivided into 3 parts: period 1 from day 1 until the first dose of ketoconazole (on day 5); period 2 from first dose of ketoconazole until concomitant administration of panobinostat with ketoconazole (on day 8 ) and period 3 from the time point of this combination until day 15 .

Most patients (12/14 patients, $85.5 \%$ ) had at least one adverse event during the core phase of the study. Overall, the most frequently reported AEs regardless of relationship to study drug were diarrhea (reported in 5 patients), anorexia (4) vomiting (4), nausea (3), hypophosphatemia (3), myalgia (3) and fatigue (2). The majority of the AEs were categorized as grade 1 or grade 2 (42.9 and $35.7 \%$ of all events, respectively).

No relevant difference was seen in ECG readings across the three periods in the core phase. The highest incidence of QTc prolongation $>30 \mathrm{~ms}$ (ms) as compared with baseline was observed in 5 patients $(36 \%)$ in period 3 (concomitant panobinostat and ketoconazole), while this increase was seen in 4 patients (29\%) and 3 patients $(21 \%)$ in periods 1 (panobinostat only) and 2 (ketoconazole only), respectively. No increases in QTc $\geq 60$ ms compared with baseline were observed. In six patients $(43 \%)$, other transient ECG abnormalities were detected during period 3, while these were seen in $3(21 \%)$ patients in either period 1 or 2 (Table 3).

One possibly study drug-related serious adverse event occurred during the core phase of the study. On day 7, after commencing study drug, a patient developed a mild increase in his serum creatinine level from $1.09 \mathrm{mg} / \mathrm{dl}$ at baseline to a maximum of $1.72 \mathrm{mg} / \mathrm{dl}$. His concomitant medications were doxazosin, irbesartan and hydrochlorothiazide for preexistent hypertension. His creatinine level returned to baseline after discontinuing the diuretic. Study treatment was permanently discontinued due to this adverse event. Overall, no differences were observed among periods 1, 2 and 3 of the core phase of the trial.

Adverse events were reported in all 13 patients continuing in the extension phase. Similar safety profile was seen in the extension phase of the study, the most frequently reported AEs regardless of relationship to study drug were nausea (reported in 10 patients), hypophosphatemia (9), diarrhea (8), vomiting (8), fatigue (7), anorexia (7), whereas thrombocytopenia was only noted in the extension phase in four patients, most likely due to longer treatment duration and corresponding higher exposure in the extension phase. The spectrum of adverse events reported in this trial was comparable with the toxicity pattern from the previous trials with panobinostat $[5,6,11,12]$. Asymptomatic hypophosphatemia of grade $\geq 2$ occurred in a total of nine out of fourteen patients (64\%) during the trial. In two of these patients, hypophosphatemia grade 2 was present before start of treatment; two other patients showed it on day 5 in the core phase, before starting ketoconazole. To further explore this finding, ad hoc urine analysis to measure fractional excretion of phosphate was performed in three out of the seven patients with treatment-emergent hypophosphatemia (6 samples) during the extension phase.

The fractional excretion of phosphate ranged from 2.7 to $19.4 \%$, while the fractional excretion of phosphate exceeded 5\% in all these patients in at least one sample (in total 4 out of 6 samples) (Table 4). No baseline samples were available.

Table 4 Fractional excretion of phosphate in patients reporting hypophosphatemia in the extension phase based on ad hoc urine analysis

\begin{tabular}{llrr}
\hline & Gender, age, tumor diagnosis & $\begin{array}{l}\text { Fractional excretion } \\
\text { of phosphate in \% (study day) }\end{array}$ & $\begin{array}{l}\text { Fractional excretion } \\
\text { of phosphate in \% (study day) }\end{array}$ \\
\hline Subj nr 00010 & Female, 55 years, invasive ductal carcinoma of the breast & 2.7 (day 36) & 8.9 (day 43) \\
Subj nr 00005 & Female, 50 years, rectal adenocarcinoma & 5.3 (day 54) & 3.2 (day 61) \\
Subj nr 00007 & Male, 71 years, cystic adenoid head and neck carcinoma & 19.4 (day 70) & 19.0 (day 77) \\
\hline
\end{tabular}

Fractional excretion of phosphate: $\left\{[\text { Phosphate }]_{\text {urine }} \times[\text { Creatinine }]_{\text {plasma }} \times 100\right\} /\left\{[\text { Phosphate }]_{\text {plasma }} \times[\text { Creatinine }]_{\text {urine }}\right\}$ 
Antitumor activity

Disease stabilization for at least 3 months was achieved in $37.5 \%$ of the patients with two patients (adenoid cystic carcinoma of head and neck, and salivary gland) staying on study treatment for 333 and 899 days, respectively.

\section{Discussion}

The prevalence of polypharmacy is increasing and is an important issue with regard to potential drug-drug interactions with untoward consequences. This study aimed to determine whether co-administration of a strong CYP3A inhibitor in patients treated with panobinostat would result in a clinically relevant change in the exposure after a single dose of panobinostat. Emerging results from this DDI study with ketoconazole and a radiolabeled mass balance study [7] have shed light on potential drug-drug interaction risk with respect to panobinostat as a CYP3A substrate. Only a limited increase in $C_{\max }$ and AUC of panobinostat was seen in most of the patients, with a ratio of 1.6 and 1.8 in the presence of ketoconazole, respectively, without a change in $T_{\max }$ or half-life. In the two dose-escalation trials, these two pharmacokinetic parameters have shown to increase linearly with the dose between 20 and $<60 \mathrm{mg}[6,13]$.

A comparison of the metabolites observed in vivo with those formed in human liver in vitro and by recombinant cytochrome P450 (CYP) enzymes indicated that the contribution of oxidative metabolism pathways mediated by CYP enzymes in patients was less than the contributions by other panobinostat metabolic pathways (e.g., reduction, hydrolysis, 1- and 2-carbon chain shortening and glucuronidation) (personal communications with Heidi Einolf, Novartis). Therefore, the impact of clinical drug-drug interactions via CYP enzymes was predicted to be minor. This was confirmed in the present study showing a $<$ twofold increase in panobinostat AUC following consecutive doses of ketoconazole at $400 \mathrm{mg}$. As the $\mathrm{AUC}_{\text {inhibition }} / \mathrm{AUC}$ ratio was 1.8 , the $\mathrm{fm}_{\mathrm{CYP} 3 \mathrm{~A}}$ (fraction of panobinostat cleared by CYP3A) was estimated to be 0.4 using the following formula $\left[\mathrm{AUC}_{\text {inhibition }} / \mathrm{AUC}=1 /\left(1-\mathrm{fm}_{\mathrm{CYP} 3 \mathrm{~A}}\right)\right] \quad$ [14] indicating CYP pathways contributed $<50 \%$ to the overall metabolism of panobinostat.

In this study, the genotype status of CYP3A does not seem to be of relevance as there was no apparent difference in panobinostat levels in patients harboring $C Y P 3 A 5^{*} 1 / * 3$ compared with those with $C Y P 3 A 5^{*} 3 / * 3$ alleles. However, no conclusions can be drawn with respect to CYP3A4 as only patients with homozygous wild-type CYP3A4*1A genotype were enrolled in this trial.

The impact of inter-individual $(60 \%)$ and intra-individual $(30 \%)$ variabilities (personal communication Margaret
M Woo, Novartis) in the pharmacokinetics of panobinostat is larger than the effect of CYP3A inhibitors (Table 2), rendering individual titration of the panobinostat dose, based on the observed toxicity during treatment, of far greater relevance than the concomitant use of a CYP3A inhibitor. Close monitoring of patients would be recommended whenever chronic co-administration of a potent CYP3A4 inhibitor is unavoidable during treatment with panobinostat.

It is worth noting that although, the recommended phase II dose in patients with solid tumors is set at $20 \mathrm{mg}$ MWF each week, the increase in $C_{\max }$ and AUC of panobinostat in the presence of ketoconazole at this dose should be put in perspective as higher doses of 40 and $60 \mathrm{mg}$ are given MWF every week in patients with Hodgkin's lymphoma and acute myeloid leukemia, respectively [6, 12]. However, we should be cognizant that severe thrombocytopenia in patients with solid tumors is regarded as a DLT, whereas this is not necessarily the case in patients with acute myeloid leukemia due to the underlying disease. Thrombocytopenia was the most common grade 3/4 toxicity, occurring in 10 out of 13 patients during dose escalation, in patients with Hodgkin's lymphoma and was dose limiting in four of them [12].

Clinical evaluation in the core phase of this study was short (15 days), but no unexpected toxicity was observed due to a potential interaction between panobinostat and ketoconazole. Thrombocytopenia was present in the extension phase, when repeated doses of panobinostat $20 \mathrm{mg}$ MWF were administered, but overall the safety profile remained as expected.

QTc prolongation has drawn attention during a phase I study with continuous intravenous administration of panobinostat [12]; however, in our current study as well as in the other studies using oral panobinostat, this does not seem to be a major issue $[5,6,11,12]$. At least at 14 time points during the 15 days of the core phase of this study, ECGs were procured in order to obtain more data on a potential QTc prolongation. The mean increase at any moment during the core phase compared with baseline was below 5\%. Also during the extension phase, the only observed QTc abnormalities were increases in QTc larger than 30 and $\leq 60 \mathrm{~ms}$.

The occurrence of hypophosphatemia during treatment with HDAC inhibitors has been previously described [1517], and a recent review of toxicities reported with HDAC inhibitors mentions asymptomatic hypophosphatemia as one of the common AEs reported in single-agent vorinostat (5\%), romidepsin (8\%) and entinostat (29\%) [16].

As mild asymptomatic hypophosphatemia was also noted in this study, and in order to evaluate renal phosphate wasting, ad hoc urine analysis was performed during the extension phase and the findings were suggestive of renal 
phosphate wasting in all three tested patients as defined by a fractional excretion of phosphate $>5 \%$. Due to lack of predefined baseline and serial assessments of phosphate in urine, firm conclusions cannot be drawn from this unplanned and explorative analysis, which should be regarded as hypothesis generating. Given the occurrence of hypophosphatemia was observed during panobinostat treatment before the initiation as well as after discontinuing of ketoconazole treatment, it seems unlikely that hypophosphatemia is caused by co-administration of a CYP3A inhibitor. At present, the exact mechanism of this side effect is not known and should deserve further studies focusing on potential tubular toxicity as a result of DAC inhibition, as hypophosphatemia might be a class effect of DAC inhibitors.

In our study, patients were monitored for electrolyte serum levels and received electrolyte supplements as soon as an electrolyte deficit was noted. Similar management of asymptomatic hypophosphatemia was successfully reported in a study with MS-275, a benzamide derivative, in patients with solid tumors and lymphoma [17].

As drug-drug interactions become more and more important in the era of personalized medicine, the extent of potential interactions should be explored in the early phase of drug development. In this study, in which each patient served as their own control, a weak interaction was found between the pan-DAC inhibitor panobinostat and the CYP3A inhibitor ketoconazole. Because the interaction is weak, it may not be clinically relevant. In our opinion, the observed toxicity is not attributable specifically due to concurrent CYP3A inhibtion because a similar safety pattern was reported for the drug interaction in the evaluation phase and as a single agent in the extension phases of the study. Most patients reported gastrointestinal-related events as well as metabolism and nutrition disorders, and most adverse events were of either grade 1 or 2 . Thrombocytopenia was observed in 4 patients and neutropenia in 2 patients, when panobinostat was administered for a prolonged period of time, but not when administered with ketoconazole. However, the interpretation of the results should be taken with caution due to the variable treatment in each individual and observation duration in the extension phase.

\section{Conclusion}

Concomitant treatment with a strong CYP3A inhibitor increased $C_{\max }$ and AUC of panobinostat by 1.6- and 1.8fold, respectively. Close monitoring of patients would be recommended whenever chronic co-administration of a strong CYP3A4 inhibitor is unavoidable during treatment with panobinostat. Asymptomatic hypophosphatemia has been observed and might be a result of renal phosphate wasting; whether this is a HDACi class effect should be further explored.

Acknowledgments The assistance of Judith ter Steeg is kindly acknowledged.

Conflicts of interest Margaret M. Woo, Maria Grazia Porro, Lily Zhao, Wenkui Li and Thomas Hengelage are employees of Novartis. None of the other authors have conflicts of interest.

Open Access This article is distributed under the terms of the Creative Commons Attribution Noncommercial License which permits any noncommercial use, distribution, and reproduction in any medium, provided the original author(s) and source are credited.

\section{References}

1. Khan O, La Thangue NB (2008) Drug insight: histone deacetylase inhibitor-based therapies for cutaneous T-cell lymphomas. Nat Clin Pract Oncol 5:714-726

2. Witt O, Deubzer HE, Milde T, Oehme I (2009) HDAC family: what are the cancer relevant targets? Cancer Lett 277:8-21

3. Bolden JE, Peart MJ, Johnstone RW (2006) Anticancer activities of histone deacetylase inhibitors. Nat Rev Drug Discov 5:769784

4. Atadja P (2009) Development of the pan-DAC inhibitor panobinostat (LBH589): successes and challenges. Cancer Lett 280:233-241

5. Ellis L, Pan Y, Smyth GK et al (2008) Histone deacetylase inhibitor panobinostat induces clinical responses with associated alterations in gene expression profiles in cutaneous T-cell lymphoma. Clin Cancer Res 14:4500-4510

6. Ottman OG, Spencer A, Prince HM et al (2008) Phase IA/II study of oral panobinostat (LBH589): a novel pan-deacetylase inhibitor demonstrating efficacy in patients with advanced hematologic malignancies. In: Proceedings of the Annual Meeting American Society Hematology; abstract 958

7. Clive S, Woo MM, Stewart M, Nydam T, Hirawat S, Kagan M (2009) Elucidation of the metabolic and elimination pathways of panobinostat (LBH589) using $\left[{ }^{14} \mathrm{C}\right]$-panobinostat. In: Proceedings of the Annual Meeting American Society Oncology; abstract \# 2549

8. Shimada T, Yamazaki H, Mimura M et al (1994) Interindividual variations in human liver cytochrome P-450 enzymes involved in the oxidation of drugs, carcinogens and toxic chemicals: studies with liver microsomes of 30 Japanese and 30 Caucasians. J Pharmacol Exp Ther 270:414-423

9. Julious SA (2005) Sample size of 12 per group rule of thumb for a pilot study. Pharm Stat 4:287-291

10. O'Brien PC, Fleming TR (1975) A multiple testing procedure for clinical trials. Biometrics 35:549-556

11. Prince HM, Bishton MJ, Johnstone RW (2009) Panobinostat (LBH589): a potent pan-deaceylase inhibitor with promising activity against hematologic and solid tumors. Futur Oncol 5:601-612

12. Dickinson M, Ritchie D, Deangelo DJ et al (2009) Preliminary evidence of the pan deacetylase inhibitor panobinostat (LBH589) in refractory Hodgkin Lymphoma. Br J Haematol 147:97-101

13. Prince HM, George D, Patnaik A, et al. (2007) Phase I study of oral LBH589: a novel deacetylase (DAC) inhibitor in advanced solid tumors and non-hodgkin's lymphoma. In: Proceedings of the Annual Meeting American Society Clinicology; abstract \# 3500 
14. Shou M, Hayashi M, Pan Y et al (2008) Modeling, prediction, and in vitro in vivo correlation of CYP3A4 induction. Drug Metab Dispos 36:2355-2370

15. Mann BS, Johnson JR, Cohen MH et al (2007) FDA approval summary: vorinostat for treatment of advanced primary cutaneous T-cell lymphoma. Oncologist 12:1247-1252

16. Subramanian S, Bates SE, Wright JJ, Espinoza-Delgado I, Piekarz RL (2010) Clinical toxicities of histone deacetylase inhibitors. Pharmaceuticals 3:2571-2767
17. Gore L, Rothenberg ML, O’Bryant CL, Schultz MK, Sandler AB, Coffin D, McCoy C, Schott A, Scholz C, Eckhardt SG (2008) A phase I and pharmocokinetic study of the oral histone deacetylase inhibitor, MS-275, in patients with refractory solid tumors and lymphomas. Clin Cancer Res 14:4517-4525 